\title{
Physical activity and nutrition program for seniors (PANS): protocol of a randomized controlled trial
}

Linda Burke ${ }^{1,2^{*}}$, Jonine Jancey ${ }^{1,2}$, Peter Howat ${ }^{1,2}$, Andy Lee ${ }^{1,2}$, Deborah Kerr ${ }^{1,2}$, Trevor Shilton ${ }^{3,1}$, Andrew Hills ${ }^{4}$, Annie Anderson ${ }^{5}$

\begin{abstract}
Background: Along with reduced levels of physical activity, older Australian's mean energy consumption has increased. Now over $60 \%$ of older Australians are considered overweight or obese. This study aims to confirm if a low-cost, accessible physical activity and nutrition program can improve levels of physical activity and diet of insufficiently active 60-70 year-olds.

Methods/Design: This 12-month home-based randomised controlled trial (RCT) will consist of a nutrition and physical activity intervention for insufficiently active people aged 60 to 70 years from low to medium socioeconomic areas. Six-hundred participants will be recruited from the Australian Federal Electoral Role and randomly assigned to the intervention ( $n=300)$ and control $(n=300)$ groups. The study is based on the Social Cognitive Theory and Precede-Proceed Model, incorporating voluntary cooperation and self-efficacy. The intervention includes a specially designed booklet that provides participants with information and encourages dietary and physical activity goal setting. The booklet will be supported by an exercise chart, calendar, bi-monthly newsletters, resistance bands and pedometers, along with phone and email contact. Data will be collected over three time points: pre-intervention, immediately post-intervention and 6-months post-study.

Discussion: This trial will provide valuable information for community-based strategies to improve older adults' physical activity and dietary intake. The project will provide guidelines for appropriate sample recruitment, and the development, implementation and evaluation of a minimal intervention program, as well as information on minimising barriers to participation in similar programs.
\end{abstract}

Trial Registration: Australian and New Zealand Clinical Trials Registry ACTRN12609000735257

\section{Background}

Australia has experienced a steady increase in the proportion of older adults, with projections that $22 \%$ of the population will be aged over 60 by 2025 [1,2]. This ageing population is heavier than a generation ago, with in excess of $60 \%$ of older adults now classified as overweight or obese [3]. Overweight and obesity levels are increasing at a rapid rate worldwide while other noncommunicable diseases (NCDs) such as heart disease, type 2 diabetes and cancer are also on the rise $[4,5]$. It has been estimated that in developed countries, the cost of obesity equates with 0.7 to $2.8 \%$ of the total yearly health expenditure [6]. For example, the annual cost of

\footnotetext{
* Correspondence: L.Burke@curtin.edu.au

'School of Public Health, Curtin University, GPO Box U 1987, Perth, WA 6845, Australia

Full list of author information is available at the end of the article
}

obesity is estimated to be $\$ 21$ billion in Australia and $\$ 2.1$ billion in the state of Western Australia [7]. The increase in the prevalence of overweight and obesity is of particular concern, in view of the strong association between excess body weight and chronic health problems. It is known that as age increases physical activity declines [8], with $46 \%$ of Australians aged 60 to 75 years being insufficiently active and 33\% being completely sedentary $[1,9]$. Over the years, the physical activity levels of older Australians have reduced $[9,10]$ while their food consumption has increased [11]. This follows the worldwide trend in diet which is shifting towards an increased consumption of saturated fats, with the level of fat consumed exceeding the recommended proportion of daily energy intake [12-14].

The benefits of regular physical activity are well recognised $[5,15,16,18-21]$, regardless of body mass index 
(BMI) [22]. The greatest health improvements appear to occur when a person moves from being sedentary $(<100$ mins/week) or involved in light (1-2.9 METS) to moderate-intensity activity (>3 METS) [23]. Low-intensity aerobic exercise is typically recommended for older adults as it can be sustained for longer, results in less tiredness and injury, and therefore may result in greater energy expenditure than high-intensity exercise [24]. The Australian Government has recently developed physical activity guidelines for older Australians to help improve their health and well being. The guidelines recommend that moderate-intensity physical activity be performed for a minimum of 30 minutes on most, preferably all, days of the week [25,26]. In addition, eating adequate amounts of fruit and vegetables can provide essential nutrients for healthy tissue bolster the immune system and protect against chronic diseases [5,27-29]. The Australian Guide to Healthy Eating [30] recommends between four to seven 75 gram serves of vegetables and two to three 150 gram serves of fruit for adults aged over 60 years. In addition, both dietary fat and refined carbohydrate should be reduced to achieve appropriate balance in macronutrient intake necessary for an acceptable body weight [31].

Maintaining adequate levels of physical activity [32] and sustaining an appropriate diet [33] are important public health goals to address obesity and to minimise the adverse physiological changes [34] associated with ageing. However, there remains a need for systematic assessment of dissemination strategies to improve health outcomes $[35,36]$, recognising that older people are a heterogeneous group that would benefit from interventions to suit their personal needs and circumstances [37]. The design of interventions needs to be rigorous [38] with large samples and longer time frames $[39,40]$. Additionally, home-based nutrition and physical activity programs for older adults may reduce future costs to health care [37]. This paper describes the protocol of a randomised controlled trial that aims to improve the physical activity and nutrition behaviours of insufficiently active people aged 60 to 70 years.

\section{Methods/Design}

\section{Study design}

This project will consist of the development, implementation and evaluation of a physical activity and nutrition intervention. The program is designed to increase physical activity levels, enhance nutritional intake and assist in the management of body weight of insufficiently active 60-70 year-olds. It will be conducted in metropolitan Perth, the capital of the State of Western Australia. The intervention and evaluation design has been based on a large pilot project that produced encouraging results with respect to adherence and behaviour change [41].
The study will be a 12-month randomised controlled trial (RCT) (Ref Figure 1). Data will be collected from participants over three time points at pre-intervention, immediately post-intervention and at 6-months poststudy. The project protocol has been approved by the Curtin University Human Research Ethics Committee (approval number HR 186/2008).

\section{Recruitment}

A stratified random sampling procedure will be adopted to recruit participants from 60 suburbs (neighbourhoods) within the Perth metropolitan area. Selection criteria for these suburbs are: (a) comprised of at least $14 \%$ 60 year-olds and above, reflecting the State average [42]; (b) containing at least 120 adults aged 60-70, to ensure a sample size sufficient for the matching of telephone numbers to the Perth Electronic White Pages [43]; and (c) are of low or medium socio-economic status (SES) based on the Socio-Economic Index for Area (SEIFA) [44], a value derived from income, education level, employment status and skill level. Suburbs will be arbitrarily matched for low and medium levels of socioeconomic status. The suburbs will then be assigned to either the intervention group or the control group using a table of random numbers. The sample size will be $n=$ 300 for each of the intervention and control groups at baseline. This strategy is based on a previous RCT conducted by the research group that successfully recruited a similar study sample [45].

Using the Federal Electoral Roll (FER), 7200 potential participants in total will be randomly drawn from the 60 Perth suburbs, with the aim to recruit 15 participants per suburb. Participants need to be: (a) "insufficiently active", i.e. not achieving 30 minutes of moderate-intensity physical activity on at least 5 days per week [25]; (b) aged 60 to 70 years; (c) healthy to the extent that participation in a low-stress physical activity and nutrition program would not place them at risk; (d) not to have taken part in any research studies that involve exercise or nutrition within the last five years; and (e) not to be on any special diet.

\section{Procedure}

The Survey Research Centre at Curtin University will match telephone numbers of the 7200 names from the FER to the Perth Electronic White Pages prior to making the initial contact. It is anticipated that the matching will yield an $80 \%$ success rate [45]. During the initial contact, the purpose of the study will be explained and the caller will determine whether the individual meets the selection criteria. Participants who give verbal consent will be assigned to an intervention or control group. A self-completion questionnaire will then be sent to them, along with an explanatory cover letter and 


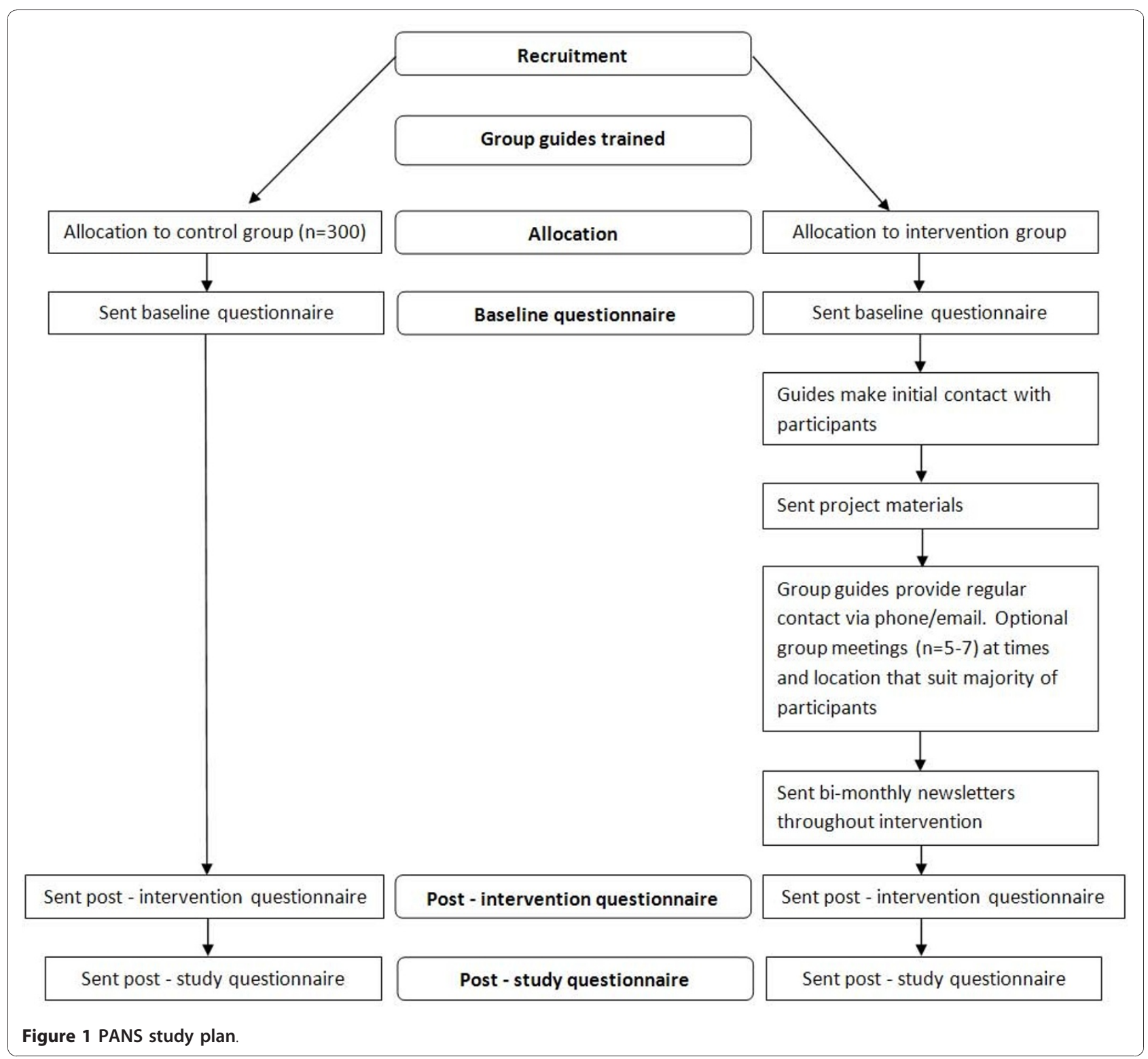

self-addressed envelope for returning the questionnaire. Participants will be advised to complete the Physical Activity Readiness Questionnaire and to furnish a medical clearance if deemed necessary before commencing the program.

\section{Intervention implementation strategies}

\section{Staff training}

Senior university Health Science students with expertise in physical activity, nutrition and health promotion will be recruited as "Guides". Potential Guides will undergo screening for suitability and intensive training; receive a comprehensive Guide's manual of dietary and physical activity guidelines; receive regular support via email and phone contact from the project coordinator; and be awarded a certificate upon completion of the training. The aim is for Guides to follow the successful New Zealand 'Green Prescription Program' $[21,46,47]$. They will be responsible for coordinating regular group meetings; phone/email contacts with participants; and be accessible for information sharing and answering questions. They will be supervised by an accredited Dietitian and a Human Movement Specialist.

\section{Provision of resources and instructional materials to participants}

The intervention group participants will receive a booklet designed to motivate them to improve their levels of physical activity and their nutrition, through goal setting. The booklet which has been updated from a pilot project $[41,48,49]$; will be supported by additional 
written materials including an interactive calendar and exercise chart. The intervention group will also be provided with a resistance band to perform the exercises described in the program, and a pedometer to monitor walking and to record the number of daily steps. A bimonthly newsletter will reinforce the key messages.

\section{Follow-up and support}

The intervention group will be allocated Guides who will conduct suburban-based group meetings (available to those who can attend) and monitor the progress of their group participants. Each Guide will supervise and support participants in one or two suburbs $(n=10$ to 20 ). They will contact their participants via phone (or email if preferred). The Guides will make three prearranged motivational phone calls at 4, 12 and 20 weeks to give advice and individualised consultation, as well as to monitor attainment of goals and provide encouragement, support and feedback $[46,47,50]$. Guides will maintain a detailed log book of the contacts made with their allocated group of participants. To increase the likelihood of sustainability, the National Heart Foundation will provide 'Heartline', a website and 1-800 telephone number for further information. All resources are designed to support participants adoption of healthenhancing behaviours with the opportunity to access information and have questions related to physical activity and nutrition answered.

\section{Control group}

Requests to complete the self-administered questionnaires will be the only contact the control group will receive from the project staff.

\section{Outcome measures}

A self-administered questionnaire will be completed by both groups of participants at baseline (pre-intervention), 6-months (immediately post-intervention) and 12-months (6-months post-intervention). The questionnaire comprises of previously validated instruments [20], and will undergo further reliability testing prior to its initial use at baseline.

Physical activity will be measured using The International Physical Activity Questionnaire (IPAQ) [51]. IPAQ has undergone extensive reliability and validity testing in 12 countries. The instrument has acceptable measurement properties for use in many settings and is specifically designed for population-based prevalence studies of physical activity. A strength exercise question based on recommendations from the American Heart Association [32] will be included to ensure the main components of the home-based exercise program are also measured.

Dietary intake will be measured using a modified version of the Fat and Fibre Barometer [52]. A question from the New South Wales Government report on soft drinks [53] will be appended to measure frequency of soft drink consumption. Validated questions will also confirm participants' stages of change regarding fruit and vegetable consumption [54]. A question from the Western Australian Physical Activity Taskforce 2005 State-wide adult physical activity survey will be used to assess confidence to participate in at least 30 minutes of physical activity on five or more days of the week [55].

General physical and mental health will be measured by The Medical Outcomes Study Short-Form Health Survey (SF-8) [56]. SF-8 is a standard generic international instrument to assess health status and is comprised of two summary scales - the physical component summary (PCS) score and the mental component summary (MCS) score.

Social support will be measured by the Dukes Social Support Scale (DSSI) [31]. The Scale is a subjective evaluation of the type and number of social interactions and has been validated for use with older people. The instrument contains two sub-scales that measure social interaction and satisfaction and has good internal consistency (Cronbach's alpha 0.77) and test-retest scores (0.70 to 0.81$)$ [31].

A single item from the Social Support for Physical Activity questionnaire [57] (SSPA) will also be used to measure perceived levels of social support for physical activity provided by friends and family. In addition, a single question will be asked about loneliness [58] while a standard validated question [59] will be used to confirm the participants' smoking status.

Demographics will be assessed by questions on gender, age, educational level, country of birth, marital status, socioeconomic status, financial status and co-morbidities. Anthropometric measures will include self-reported height and weight, waist and hip girth. A recent study has confirmed that self-assessment measures are suitable for such studies when a correction factor is applied [60].

A sub-sample of 100 participants will be selected from the intervention group. Following self-report of their height, weight, waist and hip girths the research team will measure each of these variables. Calculations of differences between self-reported and measured data will be undertaken to identify a correction factor based on the methodology of Dhaliwal et al. [60].

\section{Process evaluation}

A brief feedback sheet will be mailed to all participants to evaluate the booklet [48]. It invites the participants to rate the booklet in terms of interesting to read, easy to understand, usefulness of advice, suitability for the age group, and the relevance of messages. Participants will also be asked to comment on specific features they 
particularly like or dislike, as well as suggestions for further improvement [61]. The calendar, exercise sheet and other program resources will also be evaluated via a similar previously utilised feedback format [48].

\section{Sample size}

This is a RCT with outcomes measured at three time points. Power calculations are based on linear mixed model and assuming 70\% complete data across the three assessments due to attrition and non-respondents. In the power analyses, effect sizes of interest are associated with the correlation coefficient (or semi-partial correlation). For the mixed regression analyses of physical activity times and metabolic equivalent tasks, a sample size of $n=600$ [150 per gender by intervention or control group] will provide sufficient power $(80 \%)$ to detect a medium effect size (accounting for approximately 16\% of the variance) for gender by age interactions at a single time point without covariate adjustment. Power to detect these same interactions in the trends (based on 3 assessments) is sufficient to detect a smaller effect, accounting for approximately $11 \%$ of the variability.

\section{Discussion}

\section{Results from the PANS study are due in mid-2011}

As the aging population increases there is an urgent need to develop sound interventions capable of making a positive change to health status with consequent reduction in pressure and cost to the health care system. This physical activity and nutrition program offers a unique approach compared to other such programs for older people previously conducted in Australia for the following reasons.

The target group will be selected from younger seniors groups (60-70 years), and low and medium SES groups rather than high SES groups. Samples will be randomly selected and actively recruited through the Australian FER, and not community volunteers recruited through advertising. The intervention will provide valuable data on the effectiveness of strategies to improve dietary intake and increase physical activity in the community. The project has been designed to evaluate the strength of combining both physical activity and nutrition in order to improve the health of seniors. The evaluation data will be collected from participants in their own communities and not in a research centre, making the program relevant to the normal population and not limited to a clinical group or setting. The project will provide guidelines for appropriate sample recruitment, and the development, implementation and evaluation of a minimal, home-based tailored physical activity and nutrition intervention program. The information gathered will be useful for minimising barriers to participation in physical activity and nutrition programs. The outcomes of the project will have significant potential benefits to the Australian community via increased physical activity and better nutrition to reduce chronic disease (and associated costs), as well as enhanced mental health and improved quality of life.

\section{Acknowledgements and funding}

This study is funded by a three-year National Health and Medical Research Council (NHMRC) grant, project number 533501. The authors are grateful to Chantel Kent, Sharryn Batt and the Group Guides for their contributions to the project.

\section{Author details}

${ }^{1}$ School of Public Health, Curtin University, GPO Box U 1987, Perth, WA 6845, Australia. ${ }^{2}$ Centre for Behavioural Research in Cancer Control, Curtin University, GPO Box U 1987, Perth, WA 6845, Australia. ${ }^{3}$ National Heart Foundation, Western Australia Division, 334 Rokeby Road, Subiaco, WA 6008, Australia. ${ }^{4}$ Institute of Health and Biomedical Innovation, Queensland University of Technology, 60 Musk Avenue, Kelvin Grove QLD 4001, Australia. ${ }^{5}$ Centre for Public Health Nutrition Research, University of Dundee, Dundee, DD1 4HN, Scotland, UK.

\section{Authors' contributions}

LB coordinated the PANS program and drafted the manuscript. JJ, PH, AL, $\mathrm{LB}, \mathrm{TS}, \mathrm{DK}, \mathrm{AH}$ and $\mathrm{AA}$ designed the study, and revised the manuscript. All authors read and approved the final manuscript.

\section{Competing interests}

The authors declare that they have no competing interests.

Received: 22 November 2010 Accepted: 6 December 2010 Published: 6 December 2010

\section{References}

1. Bauman A, Bellew B, Vita P, Brown W, Owen N: Getting Australia active: Towards better practice for the promotion of physical activity Melbourne, Victoria: National Public Health Partnership; 2002.

2. Bauman A, Sallis J, Dzewaltowski D, Owen N: Toward a better understanding of the influences on physical activity: the role of determinants, correlates, causal variables, mediators, moderators, and confounders. American Journal of Preventive Medicine 2002, 23:5-14.

3. Cameron A, Welborn T, Zimmet P, DW D, Owen N, Salmon J, Dalton M, Jolley D, Shaw J: Overweight and obesity in Australia: the 1999-2000 Australian diabetes, obesity and lifestyle study (AusDiab). Medical Journal of Australia 2003, 178:427-434.

4. Popkin BM: Global nutrition dynamics: the world is shifting rapidly toward a diet linked with noncommunicable diseases. Am J Clin Nutr 2006, 84:289-298.

5. Spring B, Schneider K, McFadden Hg, Vaughn J, Kozak A, Smith M, Moller A, Epstein L, Russell S, DeMott A, Hedeker D: Make Better Choices (MBC): Study design of a randomized controlled trial testing optimal technology-supported change in multiple diet and physical activity risk behaviors. BMC Public Health 2010, 10:586.

6. The economic burden of obesity worldwide: a systematic review of the direct costs of obesity. [http://dx.doi.org/10.1111/j.1467-789X.2009.00712.x].

7. Physical Activity Taskforce: Premier's Physical Activity Taskforce: strategic plan 2007-2011. Book Premier's Physical Activity Taskforce: strategic plan 2007-2011 Government of Western Australia; 2007.

8. Thogersen-Ntoumani C, Loughren E, Duda J, Fox K, Kinnafick F-E: "Step by Step". A feasibility study of a lunchtime walking intervention designed to increase walking, improve mental well-being and work performance in sedentary employees: Rationale and study design. BMC Public Health 2010, 10:578.

9. Sims J, Hill K, Hun S, Haralambous B, Brown A, Engel L, Huang N, Kerse N, Ory M: National physical activity recommendations for older Australians: discussion document. Book National physical activity recommendations for older Australians: discussion document Report to the Australian Government Department of Health; 2006. 
10. Saarloos D, Nathan A, Almeida O, Giles-Corti B: Physical Activity Levels of Older Western Australians. Book Physical Activity Levels of Older Western Australians 2006.

11. Bennett $S$, Magnus P, Gibson D: Obesity trends in older adults AlHW bulletin no. 12. In Book Obesity trends in older adults AlHW bulletin no. 12 Volume 42. City: Australian Government. Australian Institute of Health and Welfare; 2004:16

12. Kumanyika S, Jeffery R, Morabia A, Ritenbaugh C, Antipatis V: Obesity prevention: the case for action. International Journal of Obesity 2002. 26:425-436.

13. Kearney J: Food consumption trends and drivers. Philisophical Transactions of The Royal Society 2010, 365:2793-2807.

14. Artinian N, Fletcher $G$, Mozaffarian D, Kris-Etherton P, Van Horn L, Lichtenstein A, Kumanyika S, Kraus W, Fleg J, Redeker N, et al: Prevention Committee of the Council on Cardiovascular Nursing. Interventions to promote physical activity and dietary lifestyle changes for cardiovascular risk factor reduction in adults: a scientific statement from the American Heart Association. Circulation. Journal of the American Heart Association 2010, 406-442

15. Crespo C, Palmieri M, Perdomo R, McGee D, Smit E, Sempos C, Lee I, Sorlie P: The relationship of physical activity and body weight with allcause mortality: Results from the Puerto Rico heart health program. Annals of Epidemiology 2002, 12:543-552.

16. Christensen U, Stovring N, Schultz-Larsen K, Schroll M, Avlund K: Functional ability at 75 : is there an impact of physical inactivity from middle age to early old age? Scandinavian Journal of Medicine Science and Sports 2006 16:245-251.

17. Risk factor monitoring: Physical activity, diet and body weight: results from the 2001 national health survey. [http://www.aihw.gov.au/ publications/cvd/padbwdb01/padbwdb01.pdf].

18. Todd J, Robinson R: Osteoporosis and exercise. Postgraduate Medical Journal 2003, 79:320-323.

19. Nelson M, Rejeski W, Blair S, Duncan P, Judge J, King A, Macera C, Castaneda-Sceppa C: Physical activity and public health in older adults: recommendations from the American college of sports medicine and the American Heart Foundation. Medicine and Science in Sports and Exercise 2007, 39:1435-1445.

20. Forsen $L$, Nina Waaler $L$, Vuillemin A, Chinapaw MJM, van Poppel MNM, Mokkink LB, Willem van M, Terwee CB: Self-Administered Physical Activity Questionnaires for the Elderly: A Systematic Review of Measurement Properties. Sports Medicine 2010, 40:601-623.

21. Kolt G, Schofield G, Kearse N, Garrett N, Schluter P, Ashton T, Patel A: The Healthy Steps Study: A randomized controlled trial of a pedometerbased Green Prescription for older adults. Trial protocol. BMC Public Health 2009, 9.

22. Blair S, Kampert J, Kohl H, Barlow C, Macera C, Paffenbarger R, Gibbons L: Influences of cardiorespiratory fitness and other precursors on Cardiovascular Disease and all-cause mortality in men and women. JAMA 1996, 276:205-210

23. CATI Technical Reference Group, National Public Health Partnership: Population health monitoring and surveillance: question development background paper. Physical activity in Australia. Book Population health monitoring and surveillance: question development background paper. Physical activity in Australia 2003

24. Speck B, Looney S: Effects of a minimal intervention to increase physical activity in women. Nursing Research 2001, 50:374-378.

25. Brown WJ, Moorhead GE, Marshall AL: Choose health: Be Active:A physical activity guide for older Australians. 3 edition. Canberra: Commonwealth of Australia and repatriation Commission; 2008

26. Sims J, Hill K, Hunt S, Haralambous B: Physical activity recommendations for older Australians. Australasian Journal on Ageing 2010, 29:81-87.

27. Commonwealth Government, Department of Health and Ageing, National Health and Medical Research Council, Ministry of Health Manatu Hauora: Nutrient Reference Values for Australia and New Zealand, including recommended dietary intakes. Book Nutrient Reference Values for Australia and New Zealand, including recommended dietary intakes 2006

28. Doerksen S, Estabrooks P: Brief fruit and vegetable messages integrated within a community physical activity program successfully change behaviour. International Journal of Behavioural Nutrition and Physical Activity 2007, 4:1-10
29. Carter P, Gray LJ, Troughton J, Khunti K, Davies MJ: Fruit and vegetable intake and incidence of type 2 diabetes mellitus: systematic review and meta-analysis. BMJ 2010, 341.

30. Department of Health and Ageing: The Australian guide to healthy eating Canberra: AGPS; 1998.

31. Goodger B, Byles J, Higganbotham N: Assessment of a short scale to measure social support among older people. Australian and New Zealand Journal of Public Health 1999, 23:260-265.

32. Haskell W, Lee I, Pate R, Powell K, Blair S, Franklin B, Macera C, Heath G, Thompson P, Bauman A: Physical activity and public health: updated recommendations for adults from the American College of Sports Medicine and American Heart Association. Circulation 2007, 116:1-13.

33. NHMRC: Dietary guidelines for older Australians. Book Dietary guidelines for older Australians (Editor ed.^eds.) City: Australian Government Publishing Service; 1999.

34. King A, Sallis J: Why and how to improve physical activity promotion: Lessons from behavioral science and related fields. Preventive medicine 2009, 49:286-288.

35. Mummery K, Scholfield G, Hinchliffe A, Joyner K, Brown W: Dissemination of a community-based physical activity project: The case of 10,000 steps. Journal of Science and Medicine in Sport 2006, 9.

36. Kroeze W, Werkman A, Brug J: A systematic review of randomized trials on the effectiveness of computer tailored education on physical activity and dietary behaviour. Annals of Behavioural Medicine 2006, 31:205-223.

37. Kamp B, Wellman N, Russell C: Position of the American Dietetic Association, American Society for Nutrition, and Society for Nutrition Education: Food and Nutrition Programs for Community-Residing Older Adults. Journal of the American Dietetic Association 2010, 110:463-472.

38. McCoy M, Couch D, Duncan N: Evaluating an internet weight loss program for diabetes prevention. Health Promotion International 2006, 20:221-228.

39. Spittaels H, De Bourdeaudhuij I: Implementation of an online tailored physical activity intercention for adults in Belgium. Health Promotion International 2006, 21:311-319.

40. Conn VS, Minor MA, Burks KJ, Rantz MJ, Pomeroy SH: Integrative review of physical activity intervention research with aging adults. Journal of American Geriatrics Society 2003, 51:1159-1168.

41. Lee A, Jancey J, Howat P, Burke L, Kerr D, Shilton T: Effectiveness of a home-based physical activity and nutrition pilot program for seniors. Book Effectiveness of a home-based physical activity and nutrition pilot program for seniors Journal of Obesity; 2011, 8.

42. Australian Bureau of Statistics: Socio-Economic Indexes for Areas (SEIFA), Data only, 2006. Book Socio-Economic Indexes for Areas (SEIFA), Data only, 2006 Australian Bureau of Statistics; 2008.

43. Jancey J, Clarke A, Howat P, Lee A, Shilton T, Fisher J: A physical activity program to mobilize older people: A practical and sustainable approach. The Gerontologist 2008, 48:251-257.

44. Australian Bureau of Statistics: Census of population and housing: socioeconomic indexes for areas (SEIFA) 2006, information paper. Book Census of population and housing: socio-economic indexes for areas (SEIFA) 2006, information paper Australian Bureau of Statistics; 2008, 17.

45. Jancey J, Howat P, Lee A, Clarke A, Shilton T, Fisher J, Iredell H: Effective recruitment and retention of older adults in physical activity research: PALS study. American Journal of Health Behavior 2006, 30:626-635.

46. Elley C, Kerse N, Arroll B, Ashton T, Robinson E: Cost-effectiveness of physical activity counselling in general practice. The New Zealand Medical Journal 2004, 117:1-7.

47. Elley C, Kerse N, Arroll B, Robinson E: Effectiveness of counselling patients on physical activity in general practice:Cluster randomised controlled trial. BMJ 2003, 326:1-6.

48. Burke L, Howat P, Lee A, Jancey J, Kerr D, Shilton T: Development of a nutrition and physical activity booklet to engage seniors. BMC Research Notes 2008, 1:1-7.

49. Lee A, Burke L, Howat P, Jancey J, Leong CC, Kerr D, Shilton T: Effectiveness of a home-based PAN (physical activity and nutrition) program for seniors. Proceedings of the Ninth International Conference of the Asia-Pacific Academinc Consortium for Public Health: 21-25 November, Tokyo, Japan 2007.

50. Lee L, Arthur A, Avis M: Evaluating a community-based walking intervention for hypertensives older people in Taiwan: a randomised controlled trial. Preventive Medicine 2007, 44:160-169. 
51. Craig C, Marshall A, Sjöström M, Bauman A, Booth M, Ainsworth B, Pratt M, Ekelund U, Yngve A, Sallis J, Oja P: International Physical Activity Questionnaire: 12-Country Reliability and Validity. Medicine and Science in Sports and Exercise 2003, 35:1381-1395.

52. Wright J, Scott J: The fat and fibre barometer, a short food behaviour questionnaire: reliability, relative validity and utility. Australian Journal of Nutrition and Dietetics 2000, 57:33-39.

53. Flood V, Webb K, Rangan A: Recommendations for short questions to assess food consumption in children for the NSW Health Surveys, Centre for Public Health Nutrition, NSW. Book Recommendations for short questions to assess food consumption in children for the NSW Health Surveys, Centre for Public Health Nutrition New South Wales Government, Australia; 2005.

54. Jalleh G, Lin C, Donovan R: Evaluation of the Make Smoking History 'Sugar Sugar' campaign: Wave 23. Evaluation of the Make Smoking History 'Sugar Sugar' campaign: Wave 23 Centre for Behavioural Research in Cancer Control, Curtin University, Perth; 2009.

55. Milligan R, McCormack G, Rosenburg M: Physical activity of Western Australian Adults 2006. Results from the physical activity study. Physical activity of Western Australian Adults 2006. Results from the physical activity study Western Australian Government; 2007.

56. Saris-Baglama R, Dewey CJ, Chisholm G, Chisholm B, Plumb E, King J, Kosinski M, Bjorner JB, Ware JEJ: SF-8 ${ }^{\mathrm{TM}}$ Health Survey. QualityMetric Incorporated; 2007.

57. Courneya K, Plotnikoff R, Hotz S, Birkett N: Social support and the theory of planned behavior in the exercise domain. American Journal of Health Behavior 2000, 24:300-308.

58. Doorey J, Iredell H, Boldy D, Grenade L, Howat P, Steed L: Loneliness and social isolation in later life - prevalence and interventions. Loneliness and social isolation in later life - prevalence and interventions Curtin University, Perth; 2008.

59. Howat P, Hallett J, Kypri K, Maycock B, McManus A: Tobacco smoking in an Australian University sample and implications for health promotion. Preventive Medicine; 2010.

60. Dhaliwal S, Howat P, Bejoy T, Welborn T: Self-reported weight and height for evaluating community obesity studies. American Journal of Health Behavior 2010, 34:489-499.

61. United States Department of Health and Human Services: Making health communication programs work; a planner's guide. Making health communication programs work; a planner's guide National Institutes of Health; 1992.

\section{Pre-publication history}

The pre-publication history for this paper can be accessed here: http://www.biomedcentral.com/1471-2458/10/751/prepub

doi:10.1186/1471-2458-10-751

Cite this article as: Burke et al:. Physical activity and nutrition program for seniors (PANS): protocol of a randomized controlled trial. BMC Public Health 2010 10:751.

\section{Submit your next manuscript to BioMed Central and take full advantage of:}

- Convenient online submission

- Thorough peer review

- No space constraints or color figure charges

- Immediate publication on acceptance

- Inclusion in PubMed, CAS, Scopus and Google Scholar

- Research which is freely available for redistribution 